Dhaka Univ. J. Sci. 60(1): 31-36, 2012 (January)

\title{
LSPIHT Algorithm for ECG Data Compression and Transmission for Telemedicine Applications
}

\author{
Tahmina Zebin, Ekramul Farook, Syeda Zinath Aman and Shahida Rafique \\ Department of Applied Physics, Electronics \& Communication Engineering, University of Dhaka, Dhaka-1000, Bangladesh.
}

Received on 13. 11. 2010. Accepted for Publication on 20. 08. 2011.

\begin{abstract}
Medical image analysis and data compression are rapidly evolving fields with growing applications in healthcare services e.g. teleradiology, teleconsultation, e-health, telemedicine and statistical analysis of medical data. In this paper, a Layered Set Partitioning in Hierarchical Trees (LSPIHT) algorithm for medical data compression and transmission is presented. In the LSPIHT, the encoded bit streams are divided into a number of layers for transmission and reconstruction. Starting from the base layer, by accumulating bit streams up to different enhancement layers, medical data can be reconstructed with various signal-to-noise ratios (SNRs) and resolutions. Receivers with distinct specifications can then share the same source encoder to reduce the complexity of telecommunication networks for telemedicine applications. The algorithm is compared with other algorithms for encoding ECG data, and analysis shows that the LSPIHT attains better rate-distortion performance and low network complexity than other encoding techniques.
\end{abstract}

Keywords—-Discrete Wavelet Transform (DWT), ECG, Telemedicine, Layered Image Transmission (LIT)

\section{Introduction}

Compression of medical imagery is an important area of Biomedical Engineering. Medical image analysis and data compression are rapidly evolving fields with growing applications in the healthcare services e.g. teleradiology, teleconsultation, e-health, telemedicine and statistical analysis of medical data ${ }^{1}$. Not only it has brought a drastic change in the health care systems but also it has made the concept of tele-consultation and telemedicine a reality. For telemedicine, medical image compression (MIC) and analysis may even be more useful and can play an important role for the diagnosis of more sophisticated and complicated images through consultation of experts. The useful part of the image may be highlighted with more contrast and resolution for feature extraction and diagnosis through image enhancement and analysis. In telemedicine, videos and the medical images are transmitted through advanced telecommunication links, so the help of medical image compression to compress the data without any loss of useful information is of immense importance for faster transfer of information ${ }^{2}$. The image transmission time depends on the bandwidth and the data transfer rate, so for the optimum use of the channel bandwidth, it is necessary to transmit the medical image data in compressed form.

Now to save disk space and management overhead for different types of encoded medical data, a scalable transmission scheme can be used where an encoded bit stream is delivered in two or more layers. At the receiving ends, medical data with different SNRs and/or different resolutions are reconstructed by decoding bit streams accumulated up to different enhancement layers from the base layer. Users with various SNR and resolution requirements therefore can share the same source encoder and transmission system. Although many algorithms are effective for medical data compression ${ }^{3}$, they can not perform scalable coding. The wavelet-based embedded coding algorithms ${ }^{4,5,6}$ can be employed for realizing scalable systems.

Some of these algorithms, such as JPEG $2000{ }^{6}$ and the Set Partitioning in Hierarchical Trees (SPIHT) ${ }^{7}$ algorithm have been found to outperform many existing methods for image compression. However, the SPIHT algorithm is only SNR scalable since it always encodes and displays reconstructed data at a fixed resolution level (usually the full resolution). The JPEG2000 can be used for SNR or resolution scalable transmission. The algorithm allows the compression ratio (CR) at each layer to be pre-specified. Therefore, the SNR scalability can be attained if the bit stream is delivered in layer-resolution-component-position order.

This paper shows a layered SPIHT (LSPIHT) technique for the design of scalable transmission systems ${ }^{8}$. In the LSPIHT, the CR and resolution associated with each layer can be pre-specified before encoding. The transmitted images are reconstructed with $\mathrm{CR}$ and resolution identical to those of the highest layer accumulated by the decoder. Both the SNR and resolution scalabilities therefore can be achieved. To satisfy both CR and resolution constraints at each layer, starting from the base layer, the LSPIHT encodes one layer at a time until the design of the top layer is completed. The encoding of each layer is based on SPIHT which only covers the subbands with resolution lower than the resolution constraint of that layer. To enhance the performance of SPIHT at each layer, the encoding results of the previous layers are used. In this paper, the LSPIHT is applied to images of recorded ECGs for scalable transmission, where different layers are associated with distinct CRs and resolutions. Simulation results show that, as compared with other data compression techniques, the

$\overline{\text { *Corresponding Autoher tlzebin @ yahoo.com }}$ 
LSPIHT algorithm attains better rate-distortion performance for the encoding of each layer while demanding fewer resources and lower costs.

\section{Wavelet Transform and Scalable Transmission Systems}

\section{A. 2-D DWT(discrete wavelet transform) for 2-D LSPIHT}

Let $\boldsymbol{x}$ be an image to be transmitted over the scalable system. The dimension of $\mathrm{x}$ is assumed to be $2^{n} \times 2^{n}$. Let, $\mathbf{X}$ be the p-stage wavelet transform matrix of $\mathrm{x}$, where $p$ $\leq n$.Then, as shown in Fig. 1(a), $\mathbf{X}$ is also a $2^{n} \times 2^{n}$ matrix containing subbands $X_{L_{k}}, X_{V_{k}}, X_{H_{k}}, X_{D_{k}}$; where, $\mathrm{k}=0$,
$1 \ldots \ldots ., p-1$,each with dimension $2^{k+(n-p)} \times 2^{k+(n-p)}$. To be noted, in the wavelet transform matrix, the subbands $X_{L_{k}}$ (low-pass subbands at resolution level $k$ ), and $X_{V_{k}}, X_{H_{k}}, X_{D_{k}}$ ( where $V, H, D$ are orientation selective high-pass subbands at resolution level $k$ ), are obtained recursively from $X_{L_{k+1}}$ with $X_{L_{p}}=\mathrm{X}$, where resolution level $\mathrm{p}$ refers to the full resolution. The decomposition of $X_{L_{k+1}}$ into four sub-bands $X_{L_{k}}, X_{V_{k}}, X_{H_{k}}, X_{D_{k}}$ can be carried out using a simple quadrature mirror filter (QMF) scheme $^{9}$.
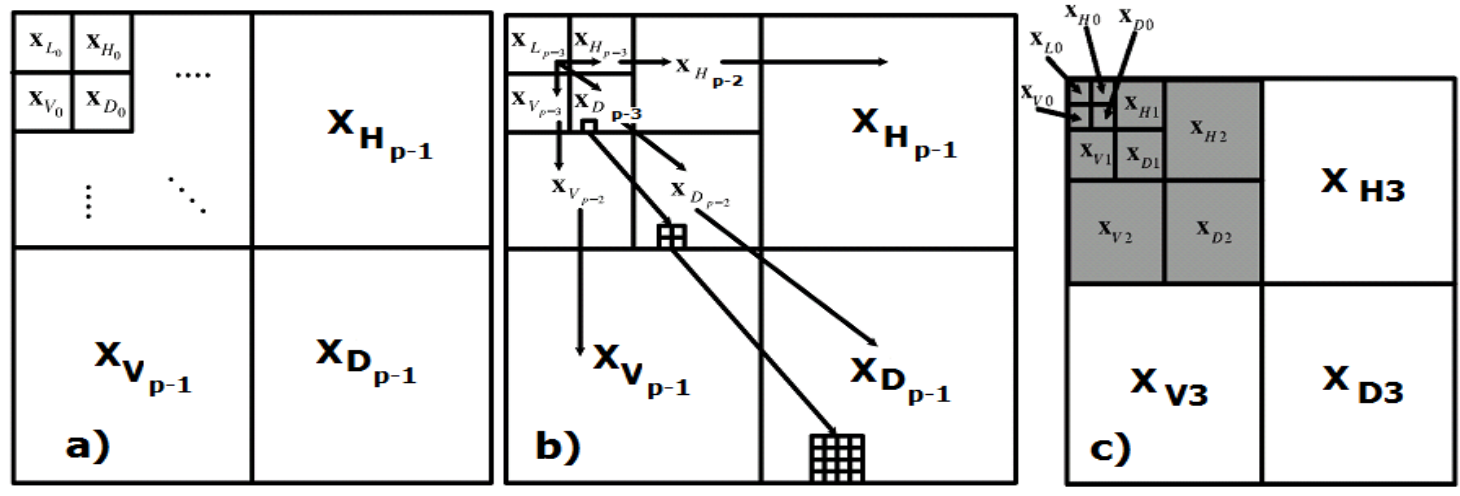

Fig. 1. a) Wavelet transform coefficients of an image $x$; b) wavelet tree; c) encoding of LIT using SPIHT.

The wavelet coefficients can be organized as a set of trees, called wavelet trees, for image coding. In the wavelet domain, with the exception of the subbands at lowest resolution level, every coefficient at a given resolution level can be related to a set of coefficients of the same orientation at the next higher resolution level. The coefficient at the lowest resolution level is called the parent, and all the coefficients at the same spatial location and of the same orientation at the next higher resolution level are called children. For the lowest resolution subband, $X_{L_{P-3}}$, in Fig. 1.(b), the parent-child relationship is defined such that each parent node has three children, one in each subband at the same resolution level and spatial location having different orientation.

\section{B. Layered Image Transmission (LIT) System}

Typical implementation of the scalable transmission systems is based on the structure of the layered image transmission (LIT) system shown in Fig. 2. In the LIT, the CR and resolution associated with each layer are desired to be prespecified. In addition, the layers are arranged in such a way that layers having lower resolution are placed in lower positions. That is, $I_{j} \leq I_{i}$; for $j \leq i$ The low-pass subband $\mathrm{X}_{\mathrm{LIi}}$ is also encoded at layer $i$. However, the encoding process at each layer is not performed independently since $\mathrm{X}_{\mathrm{LIj}}$ is a low pass sub-band of $\mathrm{X}_{\mathrm{LIi}}$, when $j \leq i$.The encoder at layer $i$ can utilize the encoding results at the previous layers to reduce the overhead for scalable transmission.

To reconstruct medical data at each layer, the decoder has to accumulate bit streams up to that layer starting from the base layer. The transmission is assumed to be lossless. The resolution of the reconstructed medical data after decoding is the resolution of the layer in the highest position among the layers decoded by the receiver.

Let the incremental rate at layer $i$ of the LIT, denoted by $\tau_{\mathrm{i}}$, be the number of bits per pixel at layer $i$. The accumulated rate $r_{i}$, can be obtained by

$$
r_{i}=r_{i-1}+\tau_{i} ; r_{0}=0
$$

Where, the incremental rate $\tau_{\mathrm{i}}$ is used to compute the Compression Ratio (CR) at layer $i$, the accumulated rate $r_{i}$ indicates the number of bits required for decoding at layer $i$. 


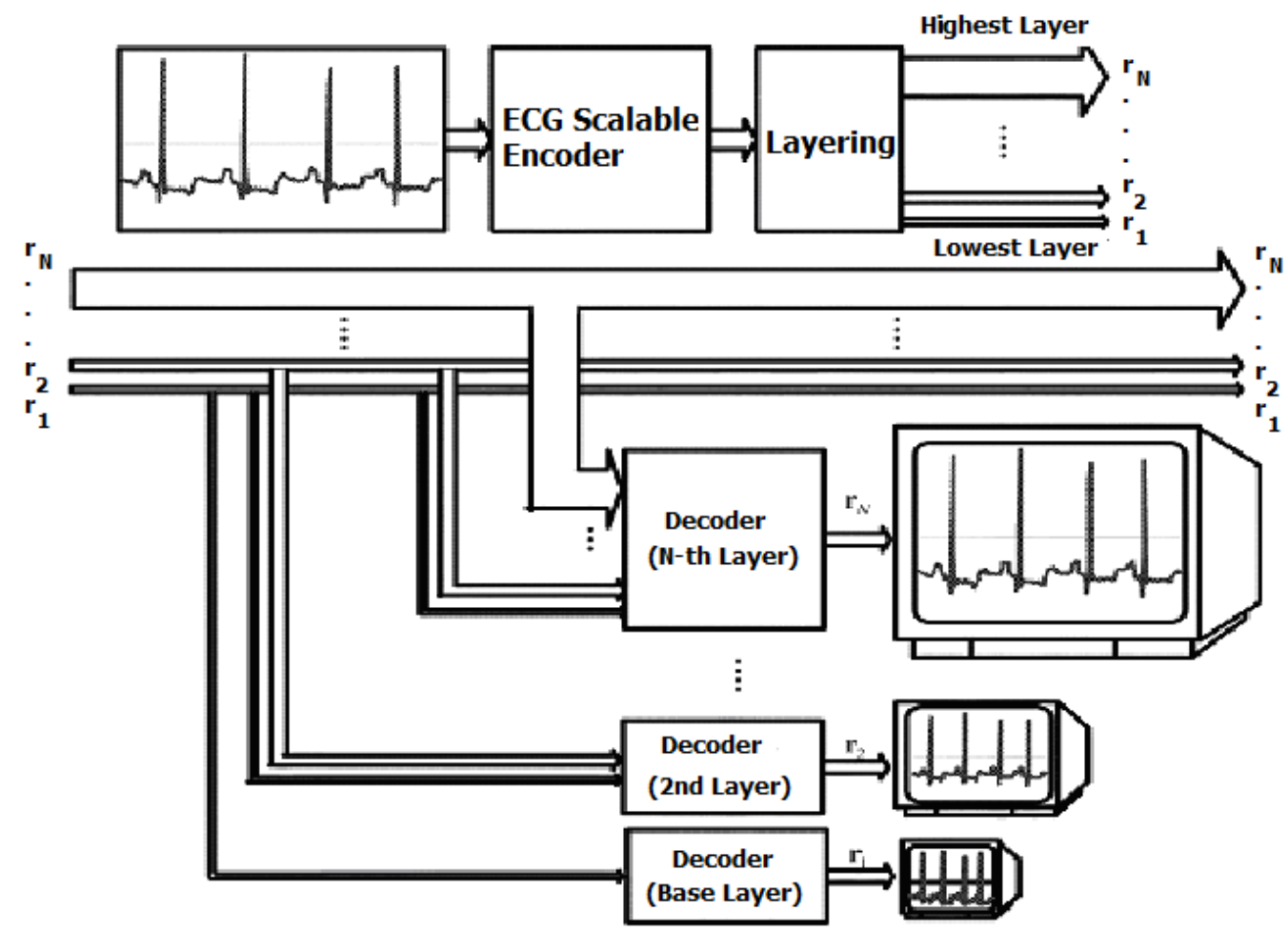

Fig. 2. Basic Structure of Layered Image Transmission (LIT) System

In the SPIHT and the JPEG2000 algorithms, the rate for encoding an image $\boldsymbol{X}$ can be pre-specified. In the LIT systems, however, the $\mathrm{X}_{\mathrm{LIi}} ; \mathrm{i}=1,2, \ldots, \mathrm{N}$ viewed as the subbands of $\boldsymbol{x}$. Since the rate allocated to these subbands can not be prespecified in the SPIHT and JPEG2000 algorithms, it may be difficult to control the incremental rate at each layer using these algorithms for the implementation of the LIT. Thus a Layered SPIHT (LSPIHT) algorithm described in the next segment could be a better solution for LIT.

\section{LSPIHT Algorithm}

The LSPIHT algorithm can be viewed as a sequence of operations using the SPIHT algorithm with one operation for each layer. Fig. 1(c) shows a simple example for the design of the LIT using the LSPIHT technique. In Fig. 1(c), the dimension of an image $x$ is assumed to be $2^{4} \times 2^{4}$ (i.e., $n=4$ ). The number of layers is $N=2$ and resolution levels associated with layer 1 and layer 2 are $I_{1}=3$ and $I_{2}=4$ respectively. Because $I_{1}=3$ the subbands $X_{\mathrm{Lo}}, X_{V K}, X_{H K}, X_{D K}$ with $k=0,1,2$ are the subbands constituting layer 1 (showed by shaded area in fig 1(c)). Similar independent encoding can be done at layer 2 with $I_{2}=4$, but this may result in large overhead.
In the SPIHT and the JPEG2000 algorithms, the rate for encoding an image $\boldsymbol{X}$ can be pre-specified. In the LIT systems, however, the $\mathrm{X}_{\mathrm{LIi}} ; \mathrm{i}=1,2, \ldots, \mathrm{N}$ viewed as the subbands of $\boldsymbol{x}$. Since the rate allocated to these subbands can not be prespecified in the SPIHT and JPEG2000 algorithms, it may be difficult to control the incremental rate at each layer using these algorithms for the implementation of the LIT. Thus a Layered SPIHT (LSPIHT) algorithm described in the next segment could be a better solution for LIT.

\section{LSPIHT Algorithm}

The LSPIHT algorithm can be viewed as a sequence of operations using the SPIHT algorithm with one operation for each layer. Fig. 1(c) shows a simple example for the design of the LIT using the LSPIHT technique. In Fig. 1(c), the dimension of an image $x$ is assumed to be $2^{4} \times 2^{4}$ (i.e., $n=4)$. The number of layers is $N=2$ and resolution levels associated with layer 1 and layer 2 are $I_{1}=3$ and $I_{2}=4$ respectively. Because $I_{l}=3$ the subbands $X_{\mathrm{Lo}}, X_{V K}, X_{H K}, X_{D K}$ with $k=0,1,2$ are the subbands constituting layer 1 (showed by shaded area in fig 1(c)). Similar independent encoding can be done at layer 2 with $I_{2}=4$, but this may result in large overhead. 


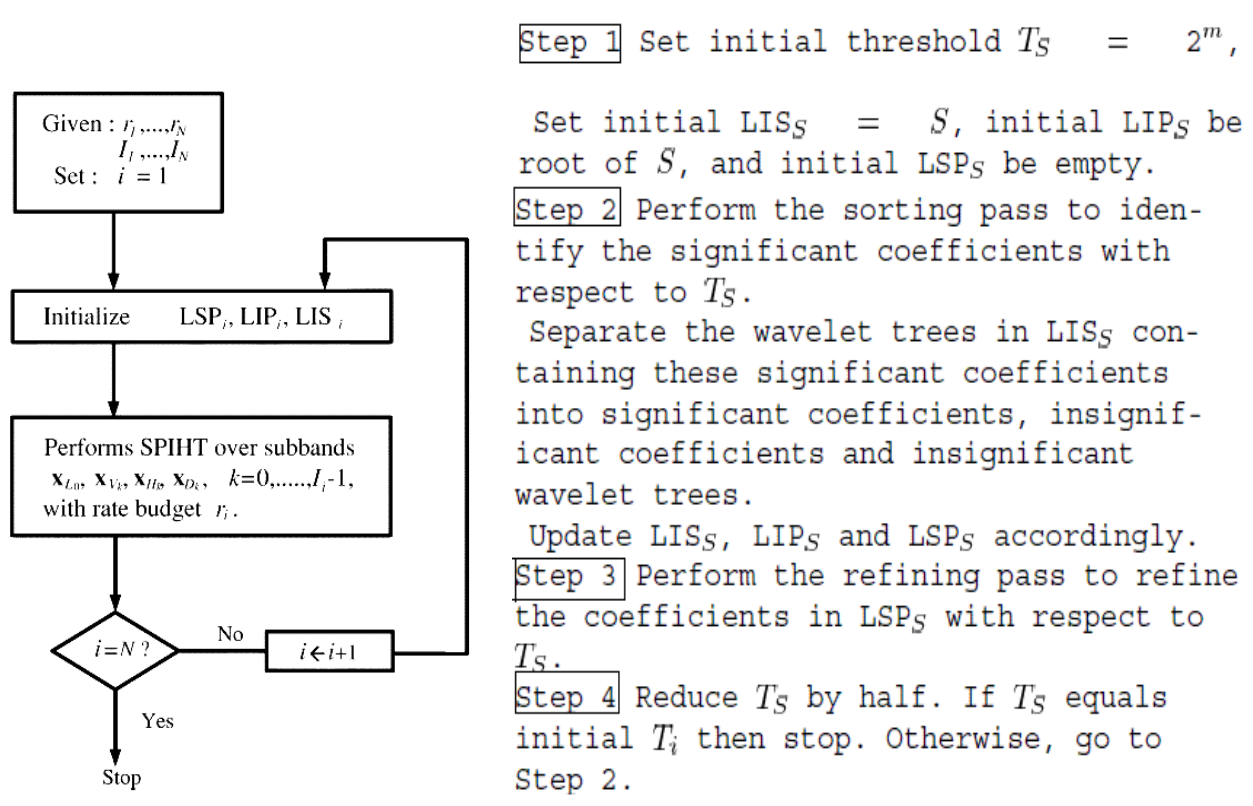

Fig. 3. Flowchart and Algorithm for the Layered Set Partitioning in Hierarchical Trees (LSPIHT) algorithm.

In contrast, using the encoding results at layer 1 for the encoding operation at layer 2 can effectively reduce this overhead.

Now to describe 2-D LSPIHT, the concept of wavelet has been recalled ${ }^{10}$. A wavelet coefficient $W$ is said to be significant for bit depth $m$ if $|\mathrm{W}| \geq 2^{m}$, otherwise it is said to be insignificant. Moreover, a wavelet tree is said to be significant for bit depth $m$ if some of its coefficients have absolute value larger than $2^{m}$.

The original SPIHT algorithm repeatedly employs a set partitioning algorithm for identifying and refining significant wavelet coefficients until the rate budget is exhausted. Each successive application of the set partitioning operation decreases the bit depth $m$ by one. For each $m$, the set partitioning operation consists of two passes: the sorting pass and the refining pass. To effectively realize these two passes, three lists of information, termed List of Significant Pixels (LSP), List of Insignificant Pixels (LIP) and List of Insignificant Sets (LIS), are maintained at any point of coding. The lists LSP and LIP contain the locations of significant and insignificant wavelet coefficients, respectively. The list LIS contains the root node of the insignificant wavelet tree. The overall flowchart and steps for the LSPIHT algorithm is shown in Fig. 3.

\section{Performance Analysis}

Table 1 shows the performance of the LIT system realized by LSPIHT for various ECGs. The ECGs are taken from the MIT-BIH arrhythmia database ${ }^{11}$ records 100, 117 and 119, sampled at $360 \mathrm{~Hz}$ and 11-bit precision.

\section{Table. 1. Performance of the ECG transmission systems realized by the LSPIHT and SPIHT algorithms}

\begin{tabular}{|c|c|c|c|c|}
\hline System & LSPIHT & T System & SPIHT & ulcast System[6] \\
\hline$i$ (layer number) & Layer 1 & Layer 2 & Layer 1 & Layer 2 \\
\hline$I_{i}($ resolution at level $i)$ & 9 & 10 & 9 & 10 \\
\hline$C R_{i}$ (Compression ratio) & $16: 1$ & $32: 3$ & $16: 1$ & $32: 3$ \\
\hline$S N R_{i}$ & 5.83 & 1.00 & 5.83 & 1.45 \\
\hline
\end{tabular}

10 min of data from each of these records has been encoded for this work. To obtain the wavelet coefficients of ECG, each record is segmented into non-overlapping and contiguous vectors having identical dimension 1024. Each vector is then independently encoded using the LSPIHT. Therefore, the full resolution level is $n=10$. The number of layers in the transmission system is $N=2$. The resolution level of layers 1 and 2 are $I_{1}=9, I_{2}=10$, and respectively. 
The performances at each layer are percent root mean square difference (PRD) and CR. For the case of ECG compression, let $\boldsymbol{X}$ be the ECG to be encoded, then the lowpass subband $X_{\mathrm{LIi}}$ is the desired ECG to be reconstructed in the layer $i$.

Let $\hat{X}_{L I_{i}}$ be the reconstructed image in the layer $i$ and $D_{i}$ be the mean squared

distance between $X_{L I_{i}}$ and $\hat{X}_{L I_{i}}$. Then the PRD in the layer

$P R D_{i}=100 \times \sqrt{\frac{D_{i}}{E_{i}}}$

where $E_{i}$ denotes the energy of $X_{L I i}$.

The CR in the layer $i$ is defined as:

$$
C R_{i}=\frac{B_{i}}{\hat{B}_{i}}
$$

Where $B_{i}$ and $\hat{B}_{i}$ are the total number of bits used for representing $X_{L I_{i}}$ and $\hat{X}_{L I_{i}}$ respectively ${ }^{12}$.

In this paper, the $C R$ at layers 1 and 2 are given by $16: 1$ and 32:3, respectively. Fig. 4 shows the original and reconstructed versions of the ECGs coded by LSPIHT at each layer. The ECGs in the layer 1 are of lower resolution and, therefore, are smaller in size. They are zoomed in/up to have same size image as in layer 2 for easy comparison. From Fig. 4, the major effect of compression is observed, especially in layer 1 , is the smoothing of the background noise. Otherwise, the features of the waveform appear to be faithfully preserved.

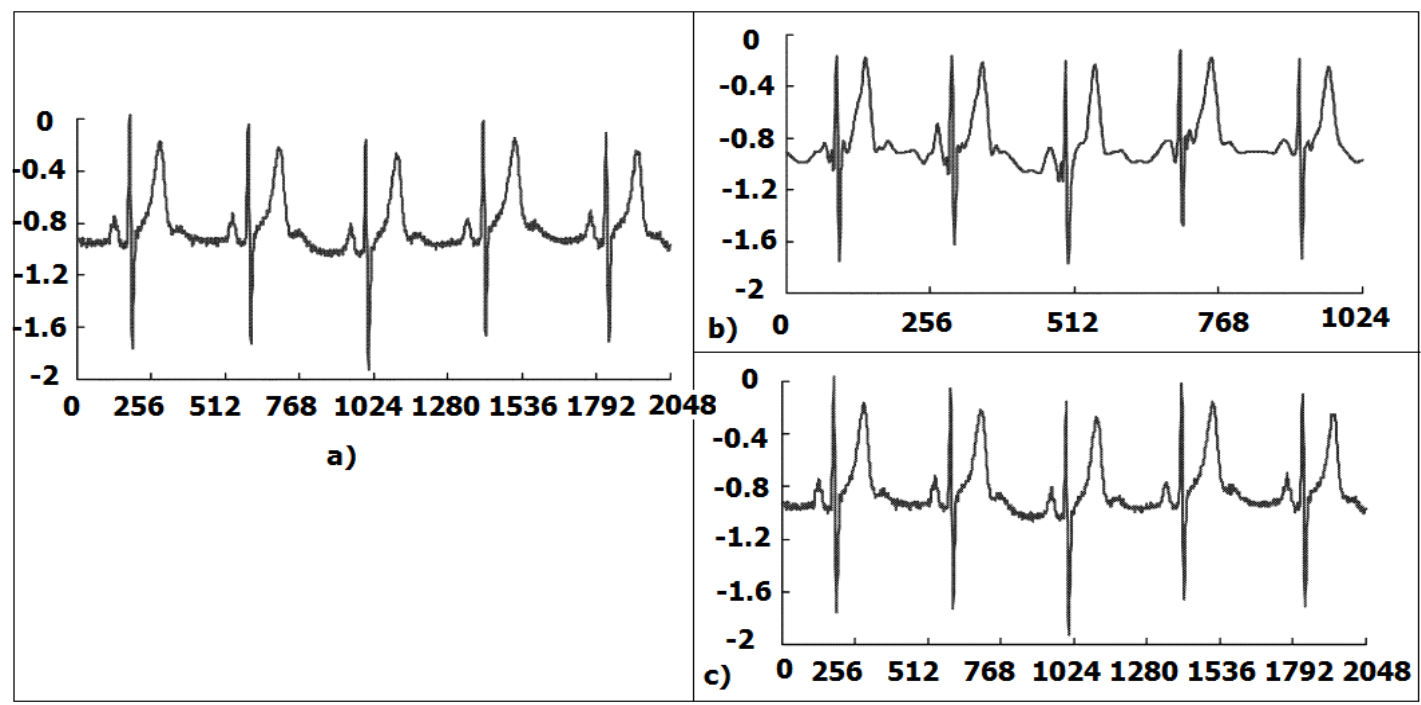

Fig. 4. Original and reconstructed ECGs of "MIT-BIH 117" at each layer of LIT shown in Table 1. a) Original ECG ${ }^{11}$. (b) Reconstructed ECG at layer 1.(c) Reconstructed ECG at layer 2.

Table 1 also shows the perfor mances of a two-layer simulcast system for encoding ECG signals. Since the JPEG2000 is used only for the image compression, only the performance of the SPIHT-based simulcast system is included in table 1 . The $\mathrm{CR}$ and resolution associated with each layer of the system is identical to those of LSPIHTbased LIT system shown in the table. Consequently, the LIT and simulcast systems have the same performance at layer 1 because both systems employ the SPIHT over the $X_{L I_{1}}$ with the same rate at that layer.

\section{Conclusion}

This paper utilizes LSPIHT technique for the scalable medical data compression and transmission. The technique allows the resolution and the compression ratio (CR or the rate) at each layer to be predefined before the design. The encoding process is then executed based on those specifications. The algorithm is based on a sequence of SPIHT algorithms and, therefore, is simple to implement. Based on the same resolution and CR at each layer, the algorithm outperforms other methods for the compression of ECG data. Because of its effectiveness and simplicity, the 
algorithm can be a good alternative for reducing the complexities and costs for realizing the communication network systems for telemedicine applications.

1. Ansari M. A., R.S. Anand, 2008, "Recent Trends in Image Compression and its Application in Telemedicine and Teleconsultation", XXXII NATIONAL SYSTEMS CONFERENCE, NSC.

2. Xiaojuan Li, G. Hu, S. Gao, 1999, "Design and implementation of a novel compression method in a teleultrasound system," IEEE Trans. On Information Tech. in Biomedicine, 3, Issue: 3, 205-213.

3. Jalaleddine S. M. S., C. G. Hutchens, R. D. Strattan, andW. A. Coberly, 1990, "ECG data compression techniques-a unified approach," IEEE Trans. Biomed. Eng., 37, 329-343.

4. Hilton M. L., 1997, "Wavelet and wavelet packet compression of electrocardiograms," IEEE Trans. Biomed. Eng., 44, 394-402.

5. Said A. and W. Pearlman, June 1996, "A new, fast, and efficient image codec based on set partitioning in hierarchical trees," IEEE Trans. Circuits Syst. Video Technol, 243-250.
6. Taubman D. S. and M. W. Marcellin, 2002, JPEG2000 Image Compression Fundamentals, Standards and Practice. Boston, MA:Kluwer.

7. Aldo Morales and Sedig Agili, 2006, "Implementing the SPIHT Algorithm in MATLAB", Department of Electrical Engineering, Penn State University at Harrisburg,

8. Wen-jyi Hwang,Ching-Fung Chine, and kuo-jung li, March 2003, "Scalable Medical Data Compression and Transmission using Wavelet Transform for Telemedicine Applications",IEEE Transactions on Information Technology in Biomedicine, 7, 1, 54-63.

9. Vetterli M. and J. Kovacevic, , 1995, Wavelets and Subband Coding. Englewood Cliffs, NJ: Prentice-Hall.

10. Djohn A., T. Q. Nguyen, and W. J. Tompkins, 1995, "ECG compression using discrete symmetric wavelet transform," Proc. 17th Int. Conf. IEEE Medicine Biology, 167-168.

11. ecg.mit.edu/

12. Metin Akay (Editor), 1997, Time Frequency and Wavelets in Biomedical Signal Processing (IEEE Press Series on Biomedical Engineering) Wiley-IEEE Press. 